\title{
INTERFACE AND SURFACE SUBSIGNALS IN PHOTOREFLECTANCE SPECTRA FOR GaAs/SI-GaAs STRUCTURES ${ }^{\ddagger}$
}

\author{
K. Jezierski, P. Sitarek, J. Misiewicz \\ Institute of Physics, Technical University \\ Wyb. Wyspiańskiego 27, 50-370 Wrocław, Poland \\ M. Panek, B. Ściana, R. Korbutowicz and M. Tlaczala \\ Institute of Electron Technology, Technical University \\ Janiszewskiego 11, 50-370 Wrocław, Poland
}

\begin{abstract}
Photoreflectance spectra were measured at room temperature for energies in the vicinity of the $E_{0}$ critical point for $p$-type as well as $n$-type doped GaAs/SI-GaAs structures. Depending on the doping concentration the existence of two photoreflectance subsignals was observed; the first one arises from the surface space charge region while the second one from the interface region. The decomposition of photoreflectance spectrum into surface and interface subsignals was based on the photoreflectance measurements carried out for different wavelengths of the laser pump beam.
\end{abstract}

PACS numbers: $68.55 .-\mathrm{a}, 71.35 .+\mathrm{z}$

\section{Introduction}

Photoreflectance (PR) is an extremely valuable technique for optical characterization of semiconductor structures $[1,2]$. In this method the modulation of the optical constants is produced by the photoinjection of electron-hole pairs by a pump light beam. When the wavelength of the laser pump beam is changed, the region where electron-hole pairs are created is shifted in the sample due to the changes in absorption of the pump beam. In turn, PR spectrum can be changed revealing the features of the structure from the different regions [3].

Recently, among many different structures, epilayers of doped GaAs have been studied extensively by means of PR method [4-6]. The aim of this paper is to present the results of PR measurements carried out for different wavelengths of the laser pump beam for the GaAs/SI-GaAs structures. Especially we are interested in the possibility of decomposition of the complex PR spectra, i.e. the spectra originated from the surface space charge region as well as from the interface.

$\ddagger$ This work has been done under the contract from the State Committee for Scientific Research (Republic of Poland) - PBZ-101-01, 370-015. 


\section{Experiment}

Doped ( $\mathrm{Si}$ and $\mathrm{Zn}$ ) GaAs epitaxial layers were grown in an atmospheric pressure, vertical MOCVD (metalorganic chemical vapour deposition) system [7]. The experimental arrangement for PR measurements was similar to that described in Ref. [7]. The coherent multiline Innova 90-6 Argon laser was used as a pump beam source. The PR spectra for GaAs/SI-GaAs structures were measured at room temperature for different wavelengths of the laser pump beam within the energy range in the vicinity of the energy gap.

\section{Results and discussion}

The obtained spectra for different $n$-type and $p$-type structures with different doping concentration can be divided into two categories. The first one (see Fig. 1) is characterized by the spectrum whose shape is independent of the wavelength of the laser pump beam. It is the case when the whole PR signal is originated only

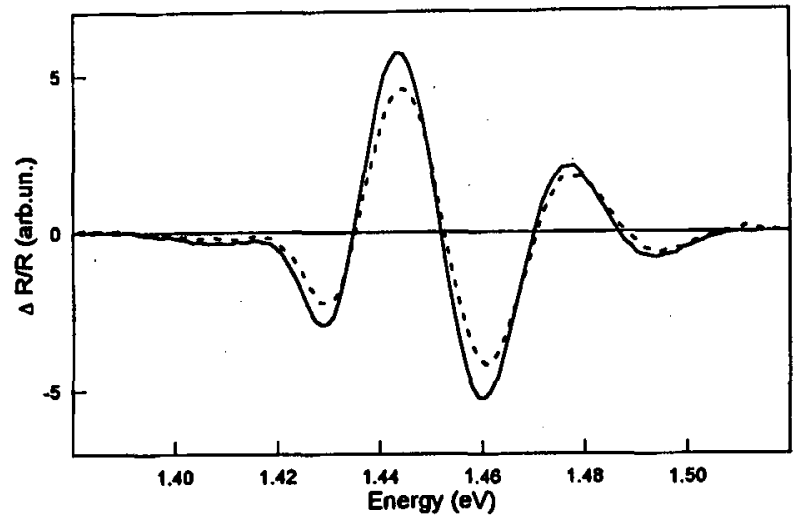

Fig. 1. PR spectra for $p$-type GaAs/SI-GaAs structure with $p=1.8 \times 10^{19} \mathrm{~cm}^{-3}$ and with the thickness of the epilayer equal to $500 \mathrm{~nm}$ measured at room temperature for the wa velength of the laser pump beam equal to $514.2 \mathrm{~nm}$ (solid line) and for $457.9 \mathrm{~nm}$ (dashed line).

from the surface space charge region or only from the interface. In Fig. 1 we can see the dumped Franz-Keldysh oscillations (FKO) for the heavily doped epilayer. The high doping concentration causes that the surface space charge region is too thin to produce any noticeable FKO and the whole PR signal is connected with the interface internal electric field. The value of this field can be estimated from the positions of FKO extrema [4] as equal to $14.3 \mathrm{kV} / \mathrm{cm}$. The critical point energy, $\mathrm{E}_{0}=1.439 \mathrm{eV}$, can be determined by means of the Kramers-Kronig analysis of the PR spectrum as peak position of the modulus of the complex photoreflectance function [8].

The second category of our PR spectra contains the spectra whose shape was changed with the change of the laser pump beam wavelength (see Fig. 2a). It should 


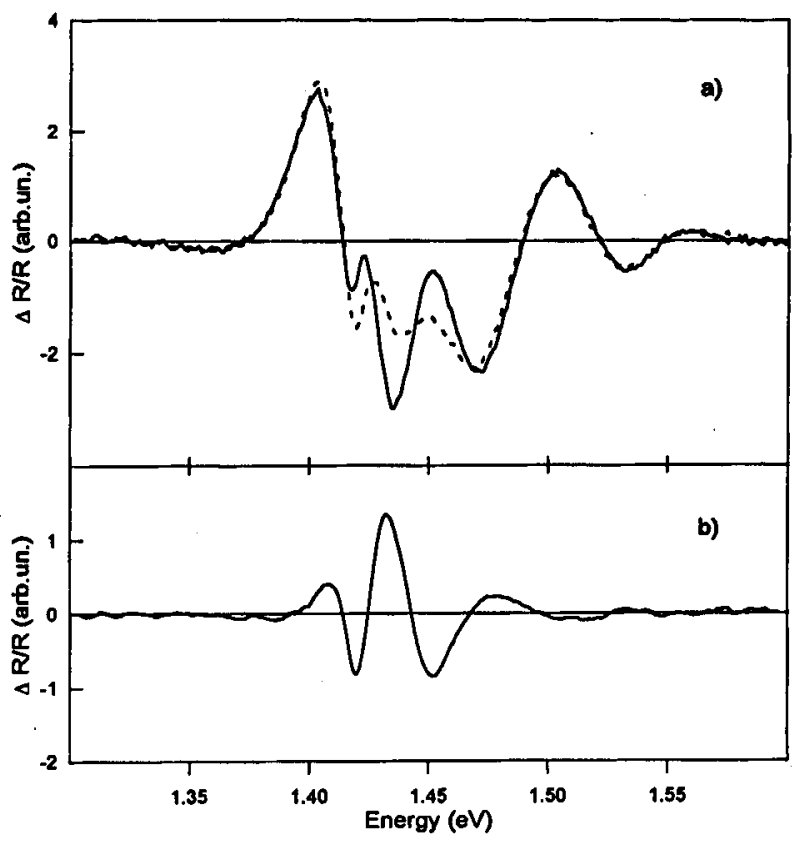

Fig. 2. PR spectra for $n$-type GaAs/SI-GaAs structure with $n=1 \times 10^{17} \mathrm{~cm}^{-3}$ and with epilayer thickness equal to $300 \mathrm{~nm}$ measured at room temperature for the wavelength of the laser pump beam equal to $514.2 \mathrm{~nm}$ (part (a) - solid line) and for $457.9 \mathrm{~nm}$ (part (a) - dashed line). In part (b) there is shown the PR subsignal from the interface determined from the two spectra shown in part (a).

be undertaken here that the power of the laser beam was kept on the same level $\left(1 \mathrm{~mW} / \mathrm{cm}^{2}\right)$. In this case each spectrum is expected to be the mixture of two subsignals: from the surface (long-period FKO) and from the interface (short-period FKO vanishing for the short wavelengths). The long-period oscillations which are visible for both wavelengths enable us to determine the built-in electric field in the surface region as equal to $37 \mathrm{kV} / \mathrm{cm}$. The determination of short-period oscillations demands the decomposition of the PR spectrum. In this case it is sufficient to subtract the spectra obtained for two wavelengths (see Fig. $2 \mathrm{~b}$ ) because the formula for PR originating from two regions for GaAs/SI-GaAs structures can be simplified to a sum of subsignals $[6,9]$ yielding

$$
\frac{\Delta R_{1}}{R}=\frac{\Delta R_{\mathrm{s}}}{R}+A \frac{\Delta R_{\mathrm{int}}}{R}, \quad \frac{\Delta R_{2}}{R}=\frac{\Delta R_{\mathrm{s}}}{R}+B \frac{\Delta R_{\mathrm{int}}}{R},
$$

where $\Delta R_{1} / R$ is the PR spectrum for the wavelength of the pump beam equal to $\lambda_{1}, \Delta R_{2} / R$ is the PR spectrum for $\lambda_{2} ; \Delta R_{\mathrm{s}} / R$ is the subsignal from the surface region whereas $\Delta R_{\text {int }} / R$ is the subsignal from the interface; $A$ and $B$ are the parameters. In our case for $\lambda_{1}=457.9 \mathrm{~nm}$ the parameter $A$ is close to zero which is connected with the fact that for such a short wavelength the penetration depth 
of light is approximately $100 \mathrm{~nm}$ while for $\lambda_{2}=514.2 \mathrm{~nm}$ it is equal approximately to $220 \mathrm{~nm}$. This difference is of a great importance for the epilayer with doping concentration $n=1 \times 10^{17} \mathrm{~cm}^{-3}$ because the width of space charge region for this layer is approximately $120 \mathrm{~nm}$. From the FKO in Fig. $2 \mathrm{~b}$ the value of the built-in electric field at interface is determined as equal to $19 \mathrm{kV} / \mathrm{cm}$.

\section{Concusions}

The PR spectra measured for different wavelengths of the laser pump beam are a valuable tool for analyzing GaAs/SI-GaAs structures. The obtained results are in very good agreement with the results obtained by means of the method based on a gradual chemical etching of the epilayer [9].

\section{References}

[1] F.H. Pollak, O.J. Glembocki, Proc. SPIE 946, 2 (1988).

[2] N. Bottka, D.K. Gaskill, R.S. Sillmon, R. Henry, R. Glosser, J. Electron. Mater. 17, 161 (1988).

[3] H. Shen, F.H. Pollak, J.M. Woodall, R.N. Sacks, J. Vac. Sci. Technol. B 7, 804 (1989).

[4] M. Sydor, J. Angelo, J.J. Wilson, W.C. Mitchell, M.Y. Yen, Phys. Rev. B 40, 8473 (1989).

[5] C.R. Lu, J.R. Anderson, D.R. Stone, W.T. Beard, R.A. Wilson, T.F. Kuech, S.L. Wright, Phys. Rev. B 43, 791 (1991).

[6] Wang Zhonghe, Pan Shihong, Huang Shuo, Zhang Cunzhou, Mu Shanming, Zhou Xlaochuan, Jian Jian, Xu Gilchang, Chen Zliongkul, J. Phys. D, Appl. Phys. 26, 1493 (1993).

[7] J. Misiewicz, P. Markiewicz, J. Rębisz, Z. Gumienny, M. Panek, B. Ściana, M. Tłaczała, Phys. Status Solidi B 183, K43 (1994).

[8] K. Jezierski, P. Markiewicz, J. Misiewicz, M. Panek, B. Ściana, R. Korbutowicz, M. Tłaczała, J. Appl. Phys., 1995, in press.

[9] K. Jezierski, J. Misiewicz, P. Markiewicz, M. Panek, B. Ściana, M. Tłaczała, R. Korbutowicz, Phys. Status Solidi A 147, 467 (1995). 